\title{
Cognitive Space-Time Transmit Pattern Design
}

\author{
Ying Sun, Zishu He, and Jun Li \\ School of Electronic Engineering, University of Electronic Science and Technology of China, Chengdu 611731, China \\ Correspondence should be addressed to Ying Sun; baobaoshu126@126.com
}

Received 2 December 2013; Accepted 2 February 2014; Published 5 March 2014

Academic Editor: Vincenzo Galdi

Copyright (C) 2014 Ying Sun et al. This is an open access article distributed under the Creative Commons Attribution License, which permits unrestricted use, distribution, and reproduction in any medium, provided the original work is properly cited.

\begin{abstract}
This paper provides a cognitive space-time (angle-Doppler) transmit pattern design method for mitigating clutter effect. This pattern takes full advantage of degrees-of-freedom (DOF) on transmit, which can preserve the maximum response for the target of interest while prenulling the mainlobe clutter on transmit, potentially simplifying processing on receiver and reducing the requirement for receiver dynamic range. The output signal-to-clutter-and-noise ratio (SCNR) can be improved significantly after applying the cognitive space-time transmit pattern to airborne-phased array radar. In addition, the traditional transmit antenna pattern and the proposed cognitive scheme are compared in terms of the probability of target detection. Simulations are conducted to demonstrate proof-of-concept.
\end{abstract}

\section{Introduction}

In the recent years, cognitive approach has been proposed and intensively investigated [1-3]. Cognitive radar is also drawing more and more attention since it was first proposed by Simon Haykin [1]. Among many tasks associated with cognitive radar, one is to adapt the transmit pattern to radar operating environment. Cognitive transmit pattern design is an innovative approach to be in pursuit of improving radar system performance $[2,4,5]$. It has been shown in [6] that this adaptive transmit technique can improve the minimum discernible velocity (MDV) - a highly desirable feature, particularly for GMTI radar.

The clutter model assumes a homogeneous ground reflectivity in existing cognitive space-time transmit pattern design methods. For a heterogeneous mixed land and sea clutter environment, however, the size and quality of the training data available are limited. Thus, it would severely limit the efficacy of these techniques $[2,5,6]$. This paper provided a cognitive space-time transmit pattern design method which applies to both homogeneous and a mixed land and sea clutter environment. The space-time adaptive processing (STAP) commonly used at receivers has been shown to provide large performance gains by jointly working across temporal and spatial domains [7-9]. Inspired by this signal processing strategy, we consider whether the space-time processing at receivers can be brought forward to the transmitters.
Our task is now to exploit the degrees of freedom (DOF) available on transmit to design transmit pattern which minimizes any energy from mainlobe clutter leaking into the main beam detection of the target of interest. Once the parameters of airborne platform including crab angle are identified, the trajectory of clutter spectrum is determined for a linear array. With the knowledge of target of interest, we provided a design method of space-time transmit pattern which places notches along the trajectory of the clutter spectrum. Thereby it reduces the amount of residual competing clutter that must be canceled in the receiver, reducing the requirement for receiver dynamic range. If the receiver enters saturation caused by a large clutter signal, the ability of the signal processor to carry out Doppler processing will be reduced and the MTI improvement factor will be degraded. To create a deep clutter notch, it is necessary to precise intrapulse and interelement modulation of the transmit signal. Advances in radar technology that permit pulse-to-pulse waveform agility make it technically feasible. It is important to note that the approach requires the high-precision measurements of the azimuth steering vector and Doppler steering vector. The proposed knowledge-aided space-time transmit pattern also shows the promise for an optimum receiver performance using a much simpler receiver structure. Simulation results in Section 4 show that the output SCNR is improved significantly after applying the cognitive space-time transmit pattern to airborne phased array radar. 
The rest of this paper is organized as follows. Section 2 describes the system model and formulates the transmit pattern design problem. In Section 3, the cognitive space-time transmit pattern design method is proposed. In Section 4, the simulation results are presented to validate the proposed method. In Section 5, some conclusions are drawn.

\section{Problem Formulation}

The system under consideration is phased array radar residing on an airborne platform. The transmitting antenna array is uniform linear arrays (ULA) consisting of $M$ elements. Each transmitting antenna radiates $L$ pulses in a coherent processing interval (CPI). We begin by briefly reviewing the airborne clutter problem in a small depression angle (far field) case. Taking forward-looking airborne phased array radar as an example, a given (and fixed) range ring consists of $N_{c}$ clutter patches distributed over $\left[-90^{\circ}, 90^{\circ}\right]$ for uniform clutter. Due to airborne platform motion, the Doppler shift of each clutter patch is given by

$$
f_{D}=\frac{2 v_{p} T_{r}}{\lambda} \cos \theta
$$

where $v_{p}$ is the speed of platform with azimuth $\theta$, wavelength $\lambda$, and pulse repetition interval (PRI) $T_{r}$. For the $c$ th $(c=$ $1,2, \ldots, N_{c}$ ) clutter patch, the transmit spatial (azimuth) steering vector and temporal (Doppler) steering vector are, respectively,

$$
\begin{aligned}
& \mathbf{a}_{T}\left(\theta_{c}\right) \\
& =\left[1, e^{-j 2 \pi d \sin \theta_{c} / \lambda}, \ldots, e^{-j 2 \pi(M-1) d \sin \theta_{c} / \lambda}\right]^{T} \in \mathbb{C}^{M \times 1} \\
& \mathbf{a}_{D}\left(f_{D_{c}}\right) \\
& =\left[1, e^{-j 2 \pi f_{D_{c}}}, \ldots, e^{-j 2 \pi(L-1) f_{D_{c}}}\right]^{T} \in \mathbb{C}^{L \times 1},
\end{aligned}
$$

where subscript $c$ indicates the index of clutter patch, $f_{D_{c}}=$ $2 v_{p} T_{r} \cos \theta_{c} / \lambda$, and $d$ is the interelement spacing of the transmit ULA.

For a deterministic point target at an angle $\theta_{t}$ and Doppler $f_{D_{t}}$, its transmit spatial (azimuth) steering vector and temporal (Doppler) steering vector are, respectively,

$$
\begin{aligned}
& \mathbf{a}_{T}\left(\theta_{t}\right) \\
& =\left[1, e^{-j 2 \pi d \sin \theta_{t} / \lambda}, \ldots, e^{-j 2 \pi(M-1) d \sin \theta_{t} / \lambda}\right]^{T} \in \mathbb{C}^{M \times 1} \\
& \mathbf{a}_{D}\left(f_{D_{t}}\right) \\
& =\left[1, e^{-j 2 \pi f_{D_{t}}}, \ldots, e^{-j 2 \pi(L-1) f_{D_{t}}}\right]^{T} \in \mathbb{C}^{L \times 1} .
\end{aligned}
$$

Extend the traditional transmit array manifold to the space-time manifold, which can be correspondingly defined as

$$
\begin{aligned}
& \widehat{\mathbf{A}}\left(\theta, f_{D}\right) \\
& =\left\{\mathbf{a}\left(\theta, f_{D}\right)=\mathbf{a}_{D}\left(f_{D}\right) \otimes \mathbf{a}_{T}(\theta),\right. \\
& \left.\quad \theta=\theta_{1}, \theta_{2}, \ldots, \theta_{N_{c}}, \quad f_{D}=f_{D 1}, f_{D 2}, \ldots, f_{D N_{c}}\right\},
\end{aligned}
$$

where $\otimes$ is the Kronecker product. The space-time transmit pattern is given by

$$
\mathbf{P}\left(\theta, f_{D}\right)=\mathbf{w}^{H} \widehat{\mathbf{A}}\left(\theta, f_{D}\right),
$$

where $\mathbf{w} \in \mathbb{C}^{M L \times 1}$ is the weight vector. The desired transmit pattern should form a notch along the trajectory of clutter spectrum while preserving the main beam response for the target of interest. Our object will thus be to choose an optimal set of complex-value space-time weights $\mathbf{w}$, so as to "prenull" the mainlobe clutter and maximize the space-time response of the target of interest. Therefore, the weight vector design can be formulated as the linearly constrained optimization problem:

$$
\begin{aligned}
& \max _{\mathbf{w}}\left|\mathbf{w}^{H} \mathbf{a}\left(\theta_{t}, f_{D_{t}}\right)\right| \\
& \text { subject to : }\left|\mathbf{w}^{H} \mathbf{a}\left(\theta_{c}, f_{D_{c}}\right)\right|=0, \quad c=1,2, \ldots, N_{c},
\end{aligned}
$$

where $\mathbf{a}\left(\theta_{t}, f_{D_{t}}\right)$ and $\mathbf{a}\left(\theta_{c}, f_{D_{c}}\right)$ for $c=1,2, \ldots, N_{c}$ are $M L \times 1$ column vectors.

\section{Knowledge-Aided Space-Time Transmit Pattern Design}

Our task is now to take full advantage of the DOFs available on transmit to design transmit pattern which minimizes any energy from mainlobe clutter leaking into the main beam detection of the target of interest. A size- $M L$ weight vector $\mathbf{w}=\left[w_{11}, w_{12}, \ldots, w_{M L}\right]$ is used to obtain the desired pattern, where $w_{m l}$ is the weight coefficient for the $m$ th transmit antenna and $l$ th transmit pulse in a CPI, as illustrated in Figure 1.

The optimization problem in (7) is equivalent to the following constrained optimization problem:

$$
\begin{aligned}
& \max _{\mathbf{w}} \mathbf{w}^{H} \mathbf{a}\left(\theta_{t}, f_{D t}\right) \mathbf{a}^{H}\left(\theta_{t}, f_{D t}\right) \mathbf{w} \\
& \text { subject to : } \mathbf{w}^{H} \mathbf{G}_{c} \mathbf{G}_{c}^{H} \mathbf{w}=0,
\end{aligned}
$$

where

$\mathbf{G}_{c}$

$$
=\left[\mathbf{a}\left(\theta_{1}, f_{D 1}\right), \mathbf{a}\left(\theta_{2}, f_{D 2}\right), \ldots, \mathbf{a}\left(\theta_{N_{c}}, f_{D N_{c}}\right)\right] \in \mathbb{C}^{M L \times N_{c}} .
$$

The relaxation of the optimization problem in (8) can be approximated to

$$
\max _{\mathbf{w}} \frac{\mathbf{w}^{H} \mathbf{a}_{t} \mathbf{a}_{t}^{H} \mathbf{w}}{\mathbf{w}^{H} \mathbf{G}_{c} \mathbf{G}_{c}^{H} \mathbf{w}}
$$




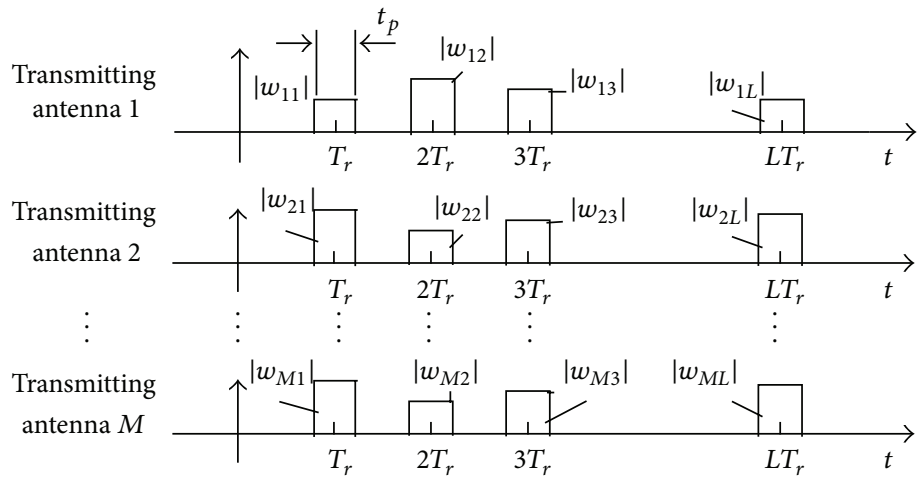

FIGURE 1: Illustration of space-time DOFs available.

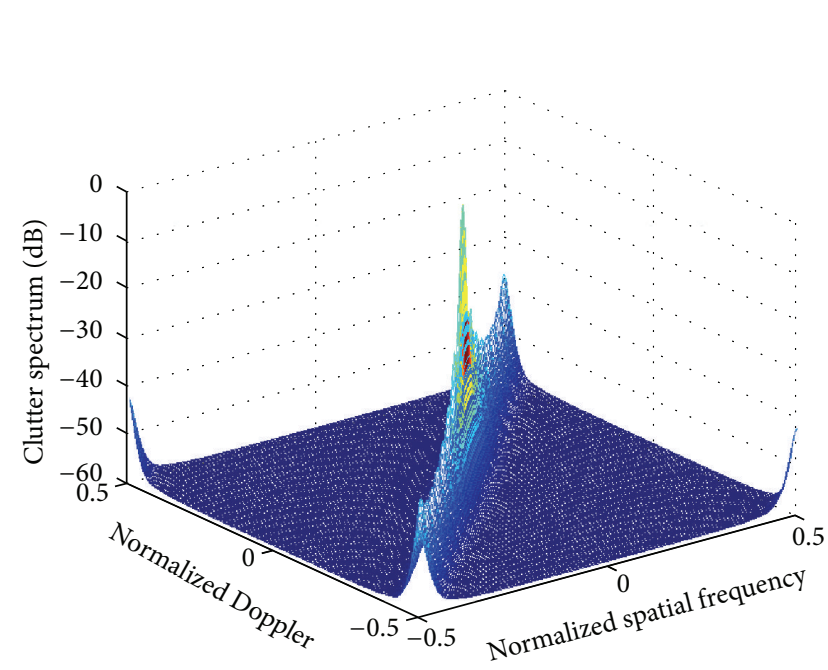

(a) The three-dimensional plot

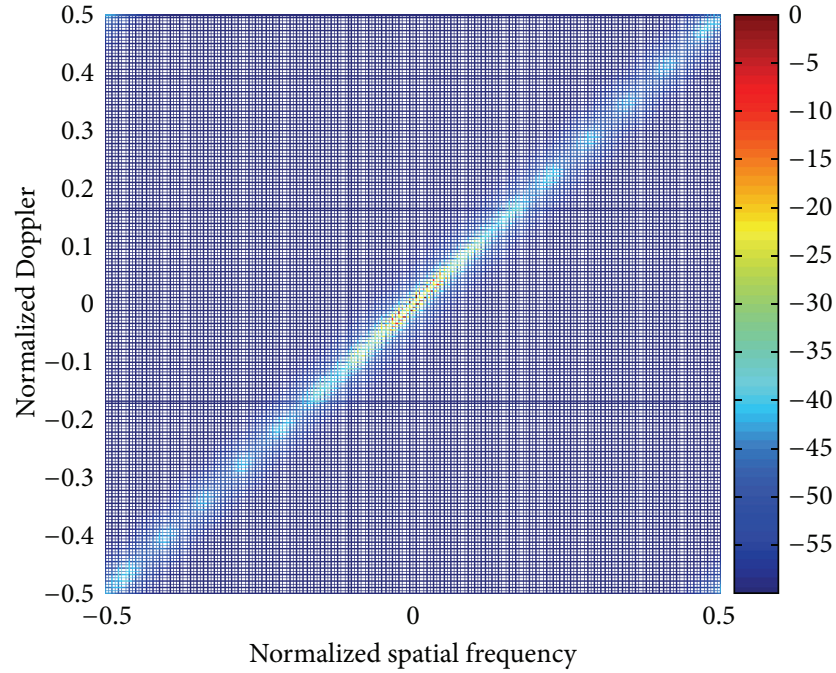

(b) Top view

FIGURE 2: Side-looking clutter spectrum.

where $\mathbf{a}_{t}=\mathbf{a}\left(\theta_{t}, f_{D t}\right)$. This is the well-known generalized Rayleigh quotient that is maximized when $\mathbf{w}$ is a solution to the generalized eigenvalue problem

$$
\left(\mathbf{G}_{c} \mathbf{G}_{c}^{H}\right) \mathbf{w}=\lambda\left(\mathbf{a}_{t} \mathbf{a}_{t}^{H}\right) \mathbf{w}
$$

with corresponding maximum eigenvalue. When $\left(\mathbf{G}_{c} \mathbf{G}_{c}^{H}\right)$ is positive definite, (10) can be converted to the following eigenvalue problem:

$$
\left(\mathbf{G}_{c} \mathbf{G}_{c}^{H}\right)^{-1}\left(\mathbf{a}_{t} \mathbf{a}_{t}^{H}\right) \mathbf{w}=\lambda \mathbf{w} .
$$

It is important to note that a small amount of diagonal loading will be employed to allow for inversion of $\left(\mathbf{G}_{c} \mathbf{G}_{c}^{H}\right)$. The solution to (11) denoted as $\mathbf{w}_{\mathrm{opt}}$ is the corresponding eigenvector of the largest eigenvalue of $\left(\mathbf{G}_{c} \mathbf{G}_{c}^{H}\right)^{-1} \mathbf{a}_{t} \mathbf{a}_{t}^{H}$. Taking the optimum eigenvector $\mathbf{w}_{\text {opt }}$ into (6) yields the transmit pattern

$$
\mathbf{P}_{\text {opt }}\left(\theta, f_{D}\right)=\mathbf{w}_{\text {opt }}^{H} \widehat{\mathbf{A}}\left(\theta, f_{D}\right) .
$$

The two-dimensional transmit pattern $\mathbf{P}_{\text {opt }}$ has the capability of notching out the mainlobe clutter while preserving the desired jointly space-time main beam response for the target of interest. Thereby it reduces the amount of residual competing clutter that must be canceled in the receiver, reducing the requirement for receiver dynamic range. If the receiver enters saturation caused by a large clutter signal, the ability of the signal processor to carry out Doppler processing will be reduced and the MTI improvement factor will be degraded. The transmit pattern also simplifies receiver structure for achieving an optimum receiver performance. The next section will validate the proposed method by simulation results.

\section{Numerical Examples}

The airborne radar system model under simulation is a narrowband 16-element ULA with half-wavelength interelement spacing. Assume that the same ULA is used for both transmission and reception and that a CPI is comprised of $L=16$ pulses.

The only knowledge used is the direction and Doppler shift of interest. Once the parameters of airborne platform including crab angle are identified, the clutter trajectory is determined. Then the desired space-time transmit pattern 


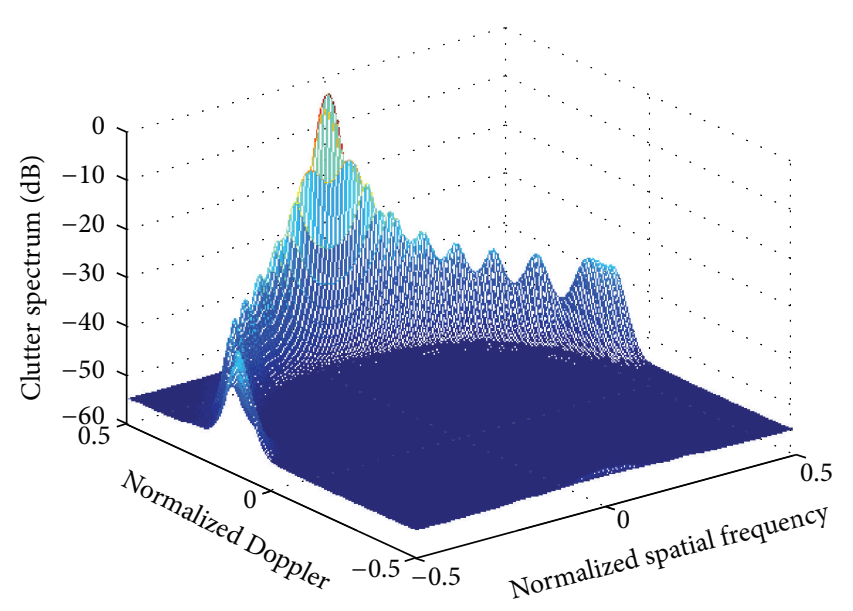

(a) The three-dimensional plot

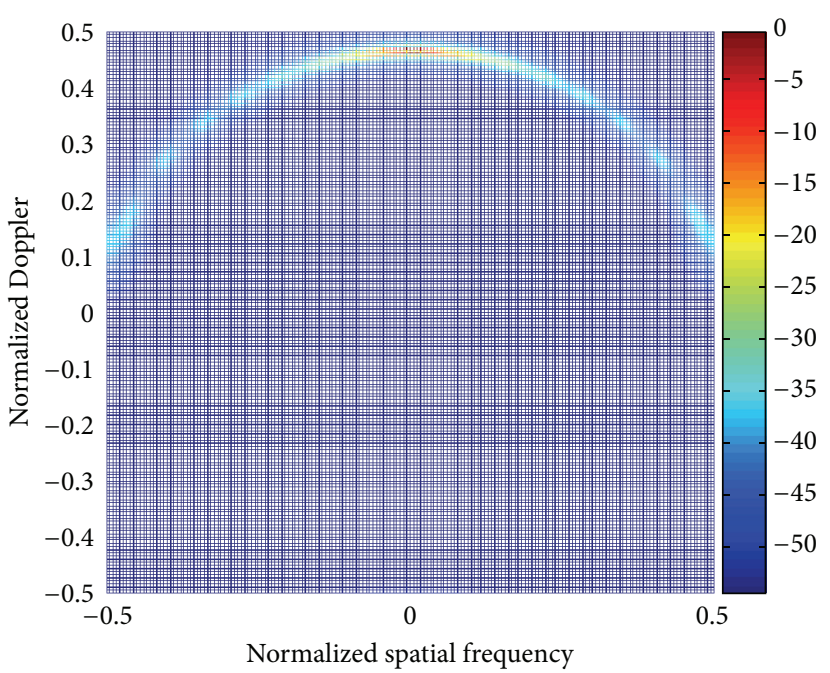

(b) Top view

FIGURE 3: Forward-looking clutter spectrum.

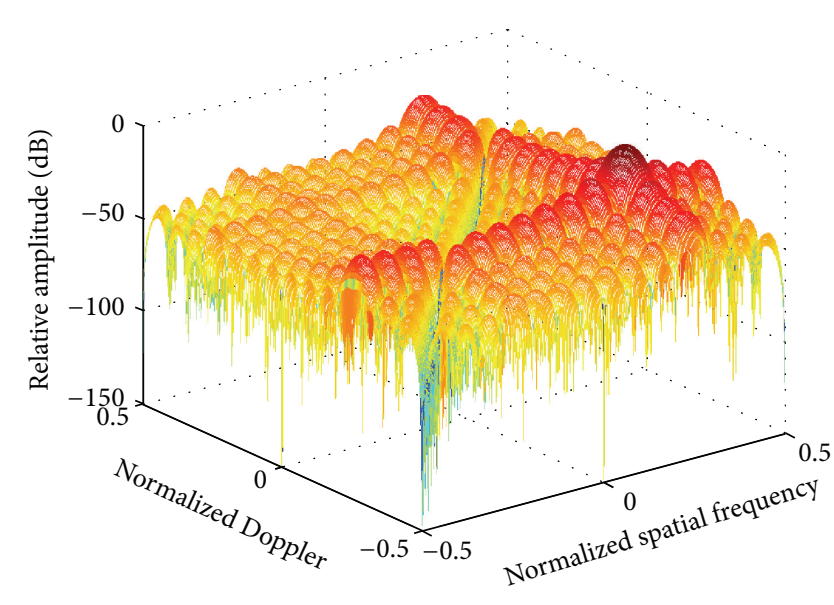

(a) The three-dimensional plot

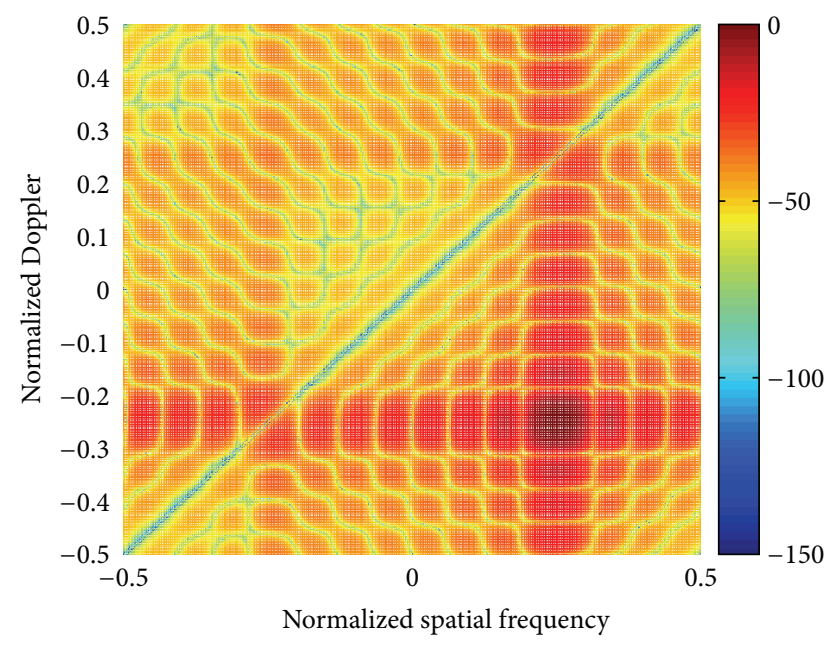

(b) Top view

Figure 4: Cognitive space-time (angle-Doppler) transmit pattern for side-looking case.

should place the notch along the trajectory of clutter spectrum and place the maximum response for the target of interest. To illustrate, we first simulate the clutter spectra in the Doppler-azimuth plane for both side-looking and forwardlooking arrays, as shown in Figures 2 and 3, respectively. The corresponding crab angles are $0^{\circ}$ and $90^{\circ}$, respectively. Figure 2(b) shows the clutter ridge for the side-looking array as a function of the normalized Doppler and normalized spatial frequency. Figure 3(b) illustrates the trajectory of clutter spectrum for a forward-looking array. The required location for space-time transmit pattern null should appear at the clutter trajectory in Figures 2 and 3.

Next, we provide the cognitive space-time transmit pattern design results. Assume the normalized spatial frequency and the normalized Doppler of the target of interest are $0.25 \mathrm{~Hz}$ and $0.25 \mathrm{~Hz}$, respectively. Other simulation parameters include wavelength $\lambda=1 \mathrm{~m}$ and pulse repetition frequency $(\mathrm{PRF}) f_{r}=2000 \mathrm{~Hz}$. The simulation results of sidelooking case are illustrated in Figure 4, where Figure 4(a) is the three-dimensional plot and Figure $4(\mathrm{~b})$ is its corresponding top view. Note that the clutter trajectory has been "prenulled," and a main beam response has been placed at the target of interest.

Figure 5 shows the cognitive space-time transmit pattern for forward-looking array, where Figure 5(a) is threedimensional plot and Figure 5(b) is its corresponding top view. Note that the space-time transmit pattern can simultaneously place a peak on the desired target with notching out the mainlobe clutter.

Next, we also provide the output SCNR simulation comparison between by using the cognitive space-time transmit pattern and by using the traditional transmit pattern under 


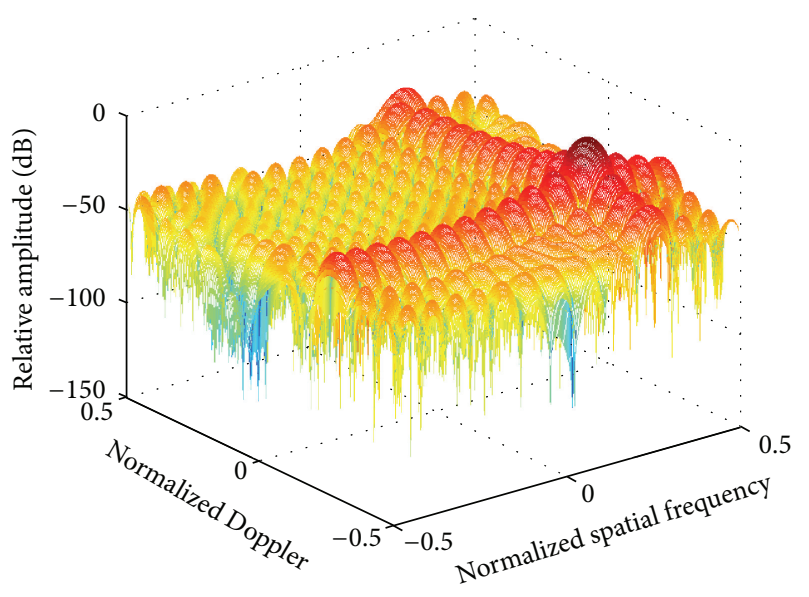

(a) The three-dimensional plot

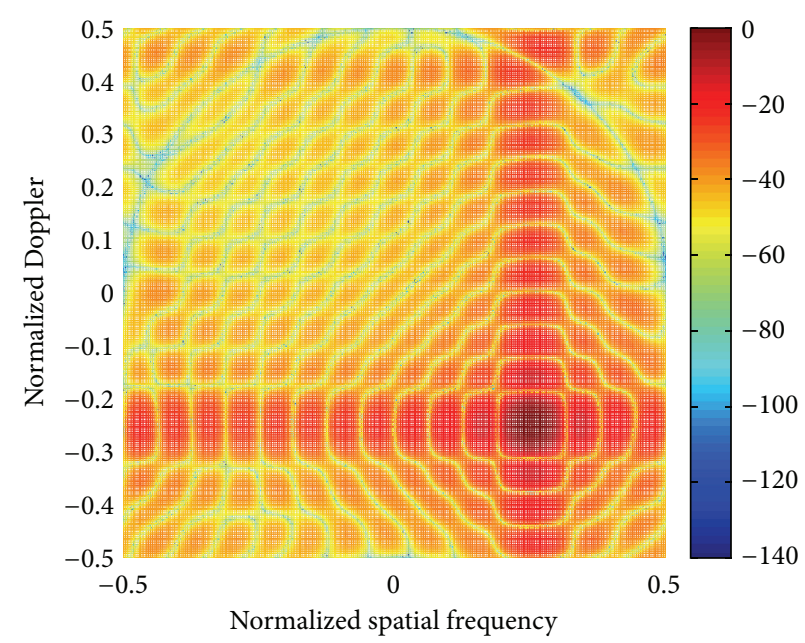

(b) Top view

FIGURE 5: Cognitive space-time transmit pattern for forward-looking case.

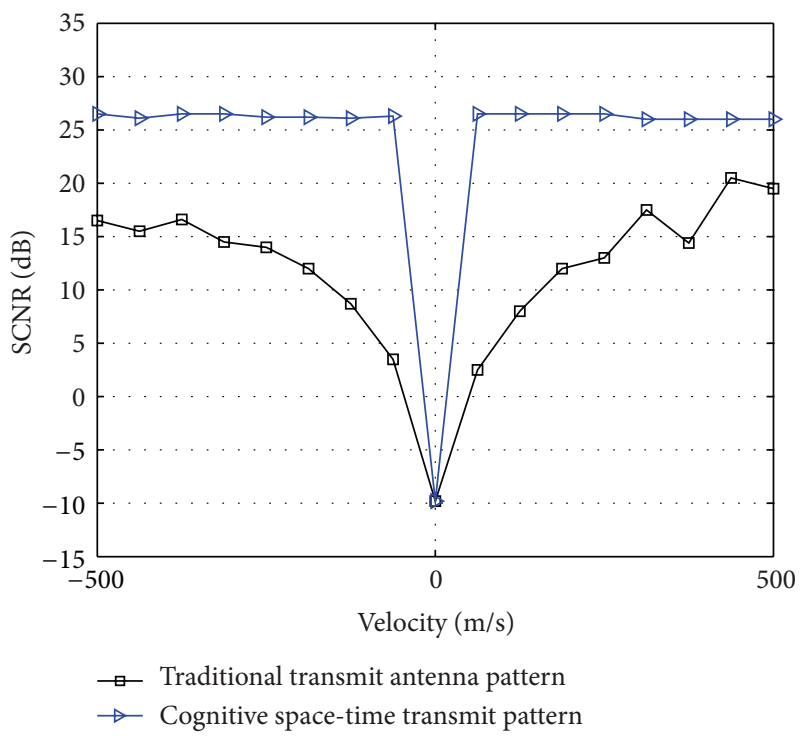

FIGURE 6: Output SCNR curve for side-looking case.

the same simulation condition. Figure 6 illustrates the impact of cognitive space-time transmit pattern applied to airborne phased array radar for side-looking case. Note that the output SCNR had significant improvement.

The impact of cognitive space-time transmit pattern applied to forward-looking airborne phased array radar is illustrated in Figure 7. Note that the output SCNR also had significant improvement.

The effect of cognitive transmit pattern is analyzed by evaluating the detection probability $\left(P_{D}\right)$ with the fixed constant false-alarm rate $\left(P_{\mathrm{FA}}\right)$. Figure 8 shows the probability of target detection when $P_{\mathrm{FA}}=10^{-6}$. The radar system using the proposed cognitive transmit pattern achieves around $13 \mathrm{~dB}$ SCR gain compared to the system which uses traditional transmit antenna pattern at $P_{D}=0.9$.

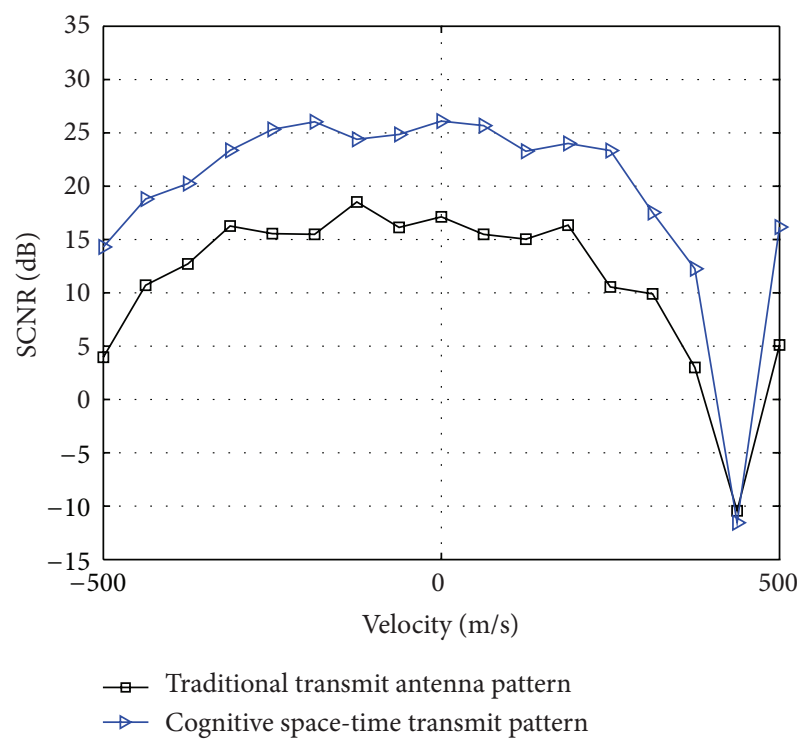

FIGURE 7: Output SCNR curve for forward-looking case.

\section{Conclusion}

This paper provided a cognitive space-time (angle-Doppler) pattern design method, which makes full use of transmit DOF. The optimum space-time transmit pattern can preserve the main beam response for the target of interest while notching out the mainlobe clutter, which can reduce the requirement of receiver dynamic range and also can simplify receiver structure for achieving the optimal receiver performance. The output SCNR can be improved significantly after applying the cognitive space-time transmit pattern to airborne phased array radar. In addition, an advantage of the radar system using the cognitive transmit pattern was demonstrated by comparing the probability of detection with traditional antenna pattern system. However, there are some 


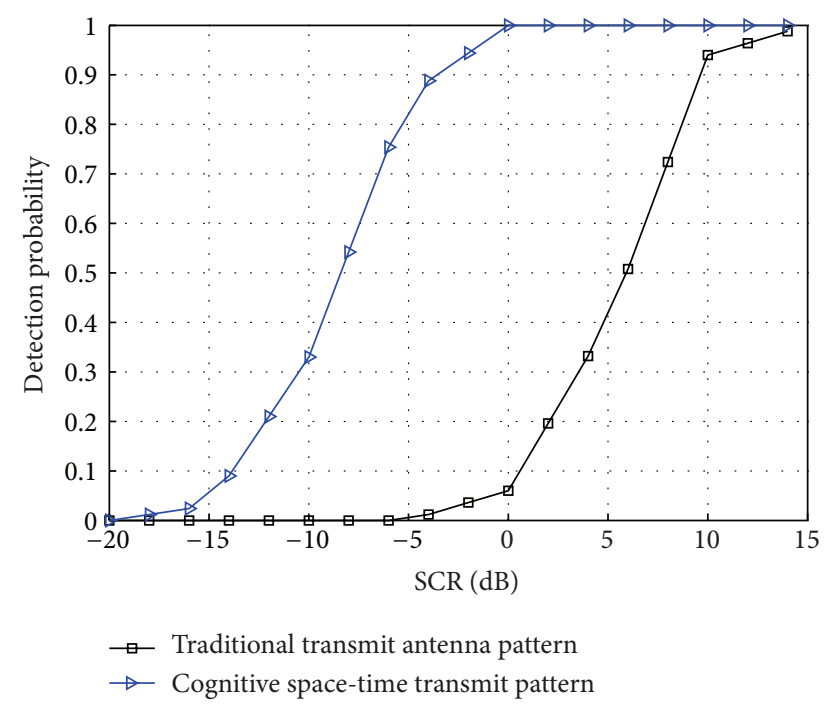

Figure 8: Probability of detection.

nonideal factors in practice. These effects of nonideal factors will be considered in the future.

\section{Conflict of Interests}

The authors declare that they have no competing financial interests.

\section{Acknowledgment}

This work is supported by the National Natural Science Foundation of China (NSFC 61032010, NSFC 61201280, and NSAF 11076006).

\section{References}

[1] S. Haykin, "Cognitive radar: a way of the future," IEEE Signal Processing Magazine, vol. 23, no. 1, pp. 30-40, 2006.

[2] J. R. Guerci, Cognitive Radar: The Knowledge-Aided Fully Adaptive Approach, Artech House, Boston, Mass, USA, 2010.

[3] A. Aubry, A. DeMaio, A. Farina, and M. Wicks, "Knowledgeaided (potentially cognitive) transmit signal and receive filter design in signal-dependent clutter," IEEE Transaction on Aerospace and Electronic System, vol. 49, no. 1, pp. 93-117, 2013.

[4] J. R. Guerci, "Theory and application of covariance matrix tapers for robust adaptive beamforming," IEEE Transactions on Signal Processing, vol. 47, no. 4, pp. 977-985, 1999.

[5] P. M. Corbell, M. A. Temple, T. B. Hale, W. P. Baker, and M. Rangaswamy, "Performance improvement using interpulse pattern diversity with space-time adaptive processing," in Proceedings of the IEEE International Radar Conference, pp. 55-60, May 2005.

[6] P. M. Corbell, M. A. Temple, and T. D. Hale, "Forward-looking planar array 3D-STAP using space time illumination patterns (STIP)," in Proceedings of the 4th IEEE Workshop on Sensor Array and Multichannel Processing, pp. 602-606, Waltham, Mass, USA, July 2006.

[7] J. R. Guerci, Space-Time Adaptive Processing for Radar, Artech House, Norwood, Mass, USA, 2003.
[8] R. Klemm, Principles of Space-Time Adaptive Processing, IET, 2002.

[9] J. Ward, "Space-time adaptive processing for airborne radar," in Proceedings of the IEE Colloquium on Space-Time Adaptive Processing, London, UK, April 1998. 

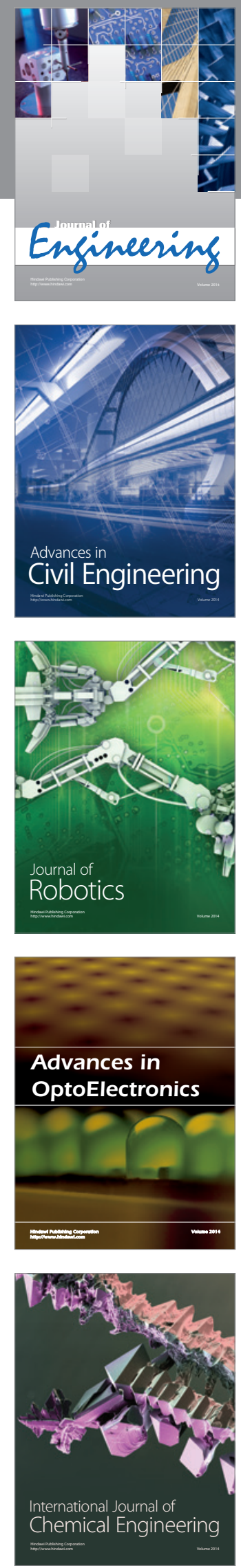

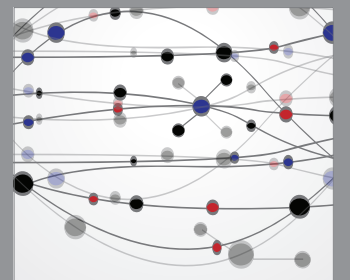

The Scientific World Journal
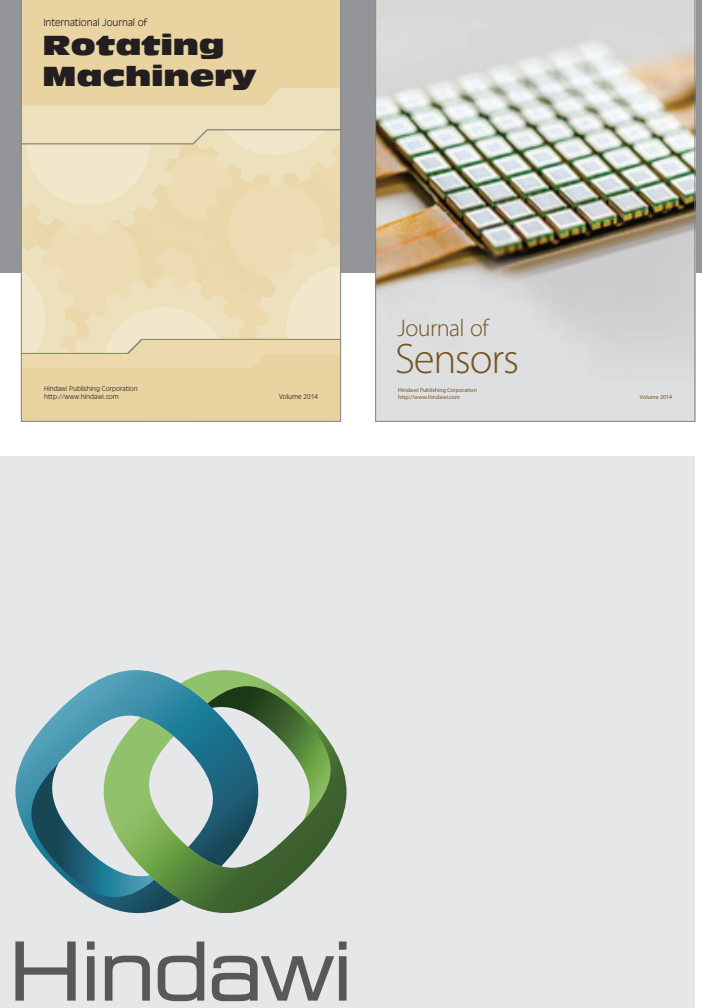

Submit your manuscripts at http://www.hindawi.com
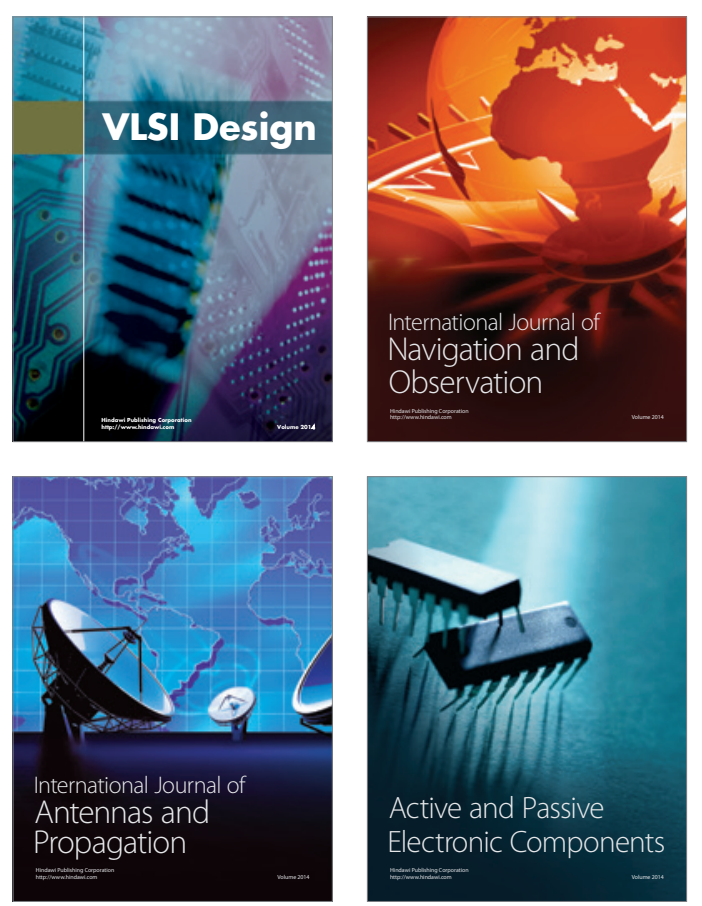
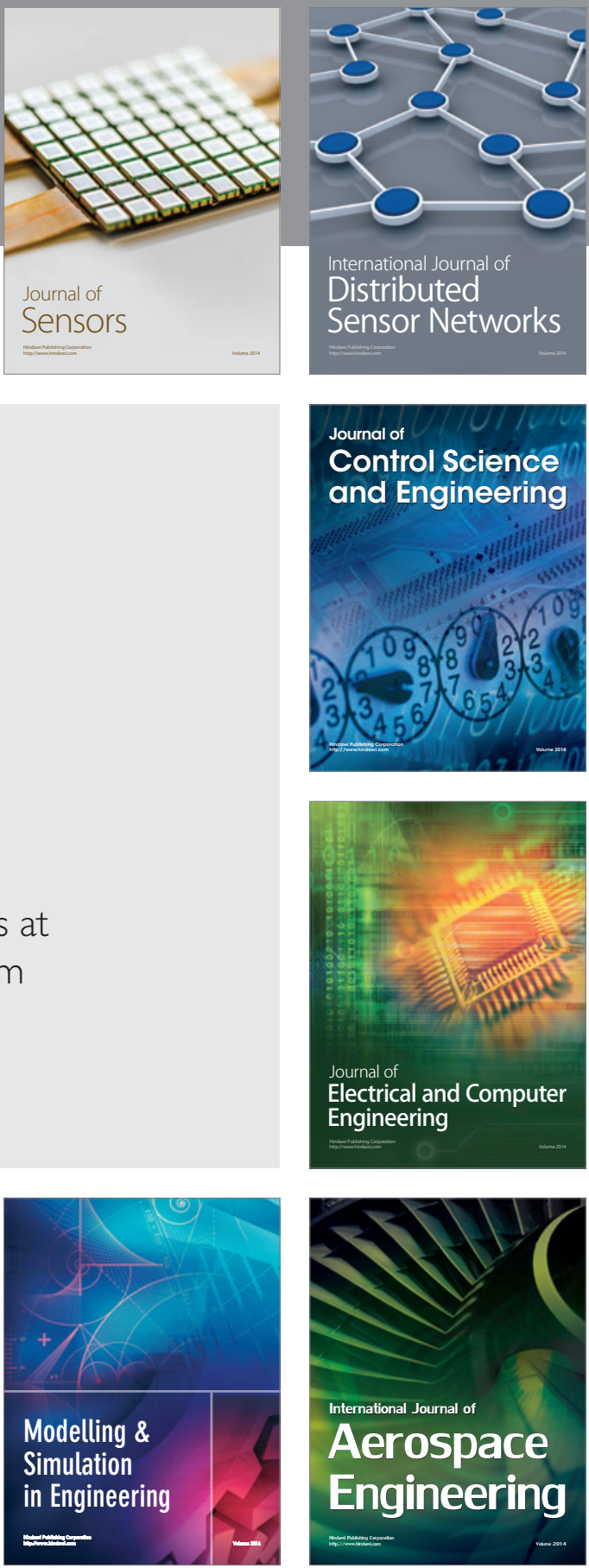

Journal of

Control Science

and Engineering
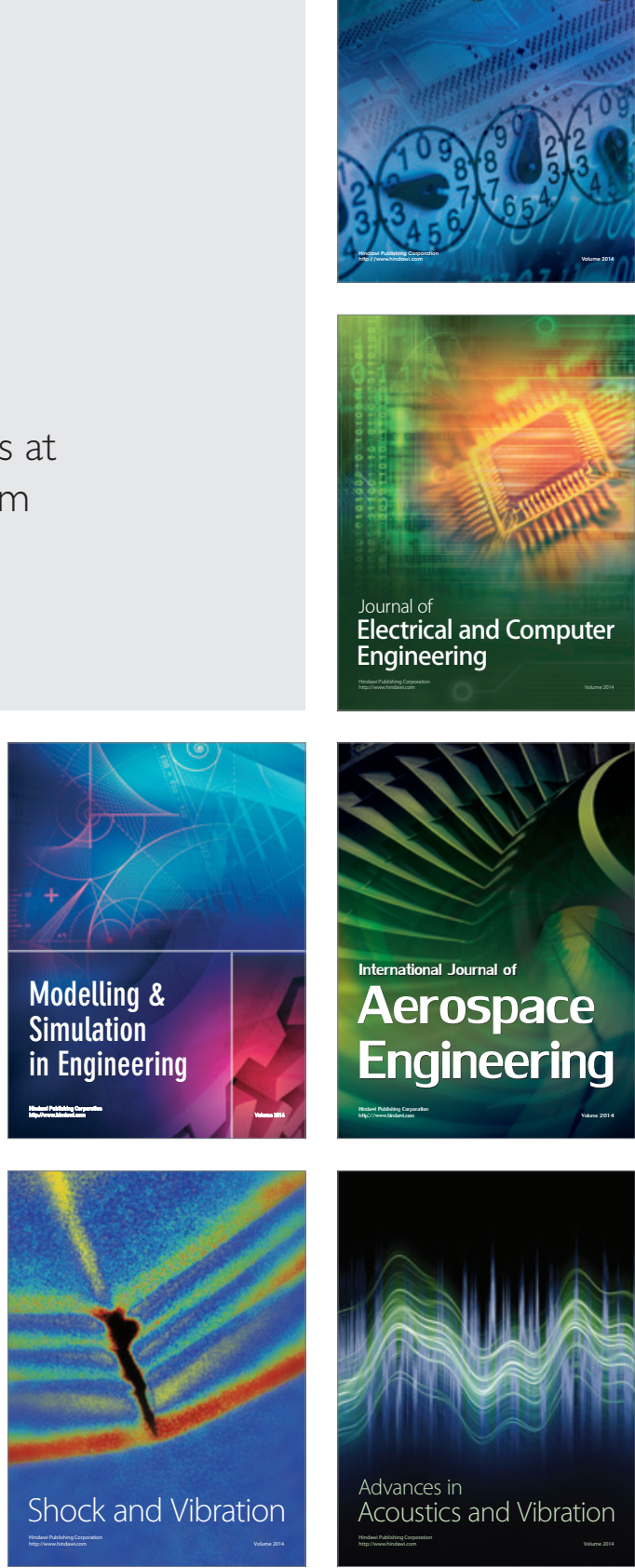